\section{APL approximations for common statistical critical values}

KENNETH J. BERRY and PAUL W. MIELKE, JR. Colorado State University, Fort Collins, Colorado

Evans and Gilfillan (1986) presented APL functions ZTAB, FTAB, CHISQ, and BINOM, which produce quantile values from $z$ scores, $F$ values, $\chi^{2}$ values, and frequencies, respectively. Quantiles of $t$ scores may be obtained from function FTAB. Statistical analyses frequently require that critical values be obtained from probability values under the $z, t, \chi^{2}$, and $F$ distributions when the required probability values are not tabled. Often such requirements arise under Bonferroni multiple comparison procedures, where in order to maintain a given level of familywise Type I error over many comparisons, the researcher may need a $t$ or $F$ critical value corresponding to a nontabled probability value. Other uses stem from the analysis of simple effects in factorial analysis of variance. This paper presents four APL functions that compute $z, t, \chi^{2}$, and $F$ critical values from upper-tail probability values. The functions are ideal for constructing tables of critical values of the $z, t, \chi^{2}, F$, and incomplete beta distributions for given probability levels.

The function that computes the $F$ values (PTOF) depends on functions for the natural logarithm of the complete beta function (LNBETA), the incomplete beta function (BETAIN), and the inverse incomplete beta function (XINBTA). These three functions may be used for other applications.

The functions are presented in Listing 1. Each of the seven functions has been written and tested in APL* PLUS/PC from STSC, Inc. IBM APL does not accept the diamond separator; enter the expressions separated by the diamonds in order from left to right. The execution times were obtained on an IBM PC-AT compatible with an 80287 internal arithmetic coprocessor.

Inverse Normal Function. Monadic function PTOZ accepts a list of one or more upper-tail probability values in its right argument and returns a vector of the corresponding approximate signed normal deviates. Thus, the result for right argument $[0.950 .050 .025]$ is $[-1.645$ $1.6451 .960]$. PTOZ uses the approximation given by Odeh and Evans (1974). Execution time for a random sample of 100 upper-tail probability values was $0.94 \mathrm{sec}$.

Inverse $\boldsymbol{t}$ Function. Dyadic function PTOT accepts a list of one or more degrees-of-freedom values in its left argument and a list of associated upper-tail probability values in its right argument. PTOT returns a vector of the corresponding approximate Student's $t$ values. Thus,

K. J. Berry's mailing address is Department of Sociology, Colorado State University, Fort Collins, CO 80523. the result for left argument [ 1520 5 and right argument $\left[\begin{array}{lll}0.95 & 0.05 & 0.025\end{array}\right]$ is [ $\left.\begin{array}{llll}-6.3138 & 2.0150 & 2.0860\end{array}\right]$.

Function PTOT uses the approximation given by Hill (1970), which utilizes an asymptotic expansion of the Cornish-Fisher type to transform normal deviates (obtained from function PTOZ) into Student's $t$ values. Execution time for a random sample of 100 upper-tail probability values and a representative sample of 100 degrees-of-freedom values was $3.57 \mathrm{sec}$.

Inverse Chi-Square Function. Dyadic function PTOC accepts a list of one or more degrees-of-freedom values in its left argument and a list of associated upper-tail probability values in its right argument. PTOC returns a vector of the corresponding approximate chi-square values. Thus, the result for left argument [ $\left.\begin{array}{lll}1 & 5 & 20\end{array}\right]$ and right argument $\left[\begin{array}{lll}0.95 & 0.05 & 0.025\end{array}\right]$ is $\left[\begin{array}{ll}3.9321 \mathrm{E}-3 & 11.0707\end{array}\right.$ 34.1696].

Function PTOC uses the approximation given by Goldstein (1973), which utilizes a 19-term polynomial to transform normal deviates (obtained from function PTOZ) into chi-square values. Execution time for a random sample of 100 upper-tail probability values and a representative sample of 100 degrees-of-freedom values was $2.85 \mathrm{sec}$.

Inverse $F$ Function. Dyadic function PTOF accepts a list of one or more paired sets of numerator-denominator degrees-of-freedom values in its left argument and a list of associated upper-tail probability values in its right argument. PTOF returns a vector of the corresponding approximate $F$ values. Thus, the result for left argument [3 $2 \varrho 1510203060]$ and right argument [0.95 0.050 .025$]$ is [4.3448E-3 2.3479 1.8152].

Function PTOF uses a simple transformation to convert inverse incomplete beta function values (obtained from function XINBTA) into $F$ values. Execution time for a random sample of 100 upper-tail probability values and a representative sample of 100 pairs of degrees-offreedom values was $38.01 \mathrm{sec}$. Although this execution time is representative for the kinds of values commonly encountered in research applications, unusual combinations (such as small numerator and large denominator degrees-of-freedom values, coupled with large probability values) may consume considerably more execution time.

Inverse Incomplete Beta Function. Dyadic function XINBTA accepts a list of one or more pairs of first and second arguments for the incomplete beta function in its left argument and a list of associated upper-tail probability values in its right argument. XINBTA returns a vector of the corresponding inverse incomplete beta function values. Thus, the result for left argument $\left[\begin{array}{l}32 \varrho 503010 \\ 20\end{array}\right.$ $24010]$ and right argument $[0.31710 .11300 .4717]$ is $\left[\begin{array}{lll}0.60 & 0.70 & 0.80\end{array}\right]$.

Function XINBTA uses initializing values (obtained from functions PTOZ and PTOC) and logarithms of the complete beta function (obtained from function 
LNBETA), and recursively obtains upper-tail probability values (obtained from function BETAIN). The algorithm given by Cran, Martin, and Thomas (1977) utilizes the Newton-Raphson method to transform the incomplete beta function values into inverse incomplete beta function values. Execution time for a random sample of 100 uppertail probability values and a representative sample of 100 pairs of positive parameter values was $36.50 \mathrm{sec}$.

Incomplete Beta Function. Dyadic function BETAIN accepts a list of one or more pairs of first and second arguments for the incomplete beta function in its left argument and a list of associated beta distribution values in its right argument. BETAIN returns a vector of the corresponding approximate upper-tail probability values. Thus, the result for left argument [ $32 \varrho 503010240$ 10] and right argument [0.60 0.700 .80$]$ is [0.3171 0.1130 0.47171 .

Function BETAIN uses the approximation given by Majumder and Bhattacharjee (1973), incorporating the improvements suggested by Cran et al. (1977), to transform beta distribution values into approximate upper-tail probability values. Execution time for a random sample of 100 beta distribution values and a representative sample of 100 pairs of positive parameter values was $6.00 \mathrm{sec}$.

Log Beta Function. Monadic function LNBETA accepts a list of one or more pairs of first and second arguments of the beta function in its right argument and returns a vector of the corresponding natural logarithms of the complete beta function. Thus, the result for right argu-

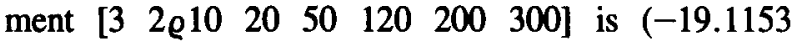
$-103.8466-337.9801]$.

Function LNBETA computes the natural logarithms of the beta function values from the logarithms of three gamma functions. Parameter values less than 100 are converted to gamma values by the APL monadic factorial function; parameter values equal to or greater than 100 are converted to gamma values by Stirling's approximation using the algorithm given by Pike and Hill (1966). Execution time for a representative sample of 100 pairs of parameter values was $1.92 \mathrm{sec}$.

\section{REFERENCES}

Cran, G. W., Martin, K. J., \& Thomas, G. E. (1977). A remark on algorithms AS 63: The incomplete beta integral; AS 64: Inverse of the incomplete beta function ratio. Applied Statistics, 26, 111-114.

Evans, S., \& GiLfillan, L. (1986). APL approximations for common statistical tables. Behavior Research Methods, Instruments, \& Computers, 18, 337-338.

Goldstein, R. B. (1973). Algorithm 451. Chi-square quantiles [G1]. Communications of the ACM, 16, 483-485.

HiLL, G. W. (1970). Algorithm 396. Student's $t$-quantiles [S14]. Communications of the ACM, 13, 619-620.

Majumder, K. L., Bhattacharjee, G. P. (1973). Algorithm AS 63. The incomplete beta integral. Applied Statistics, 22, 409-411.

ODEh, R. E., Evans, J. O. (1974). Algorithm AS 70. The percentage points of the normal distribution. Applied Statistics, 23, 96-97.

Pike, M. C., Hill, I. D. (1966). Algorithm 291. Logarithm of the gamma function. Communications of the $A C M, 9,684$.

LISTING 1

APL Functions for Approximate Critical Values of the Normal, Student's $t$, chi-square, and $F$ Distributions

$\nabla Z$ Z PTOZ P;T;C;Y;SIGN;YZ

[1] A P CARRIES PROBABILITY VALUE (S), $Z$ RETURNS $Z$ VALUE(S); FOR EXAMPLE,

[2] $A$ FOR $P+0.0250 .050 .95, Z=1.9601 .645-1.645$

[3]

[4]

[5]

[6]

[7]

[8]

$\rightarrow(\wedge /(O<F) \wedge(1>P)) / 4 \otimes$ D*'PRDBABILITY MUST BE $>0$ AND $<1 \cdot \theta \rightarrow 0$

C $4-4.53642210148 E^{-5}-0.0204231210245-0.342242088547-1$

$C \leftarrow\left[,-0.322232431088 \quad 3.8560700634 E^{-3} \quad 0.103537752850 .531103462366\right.$

$C+C, 0.5885815704950 .099348462606 *$ SIGN $41+-2 \times P>0.5$

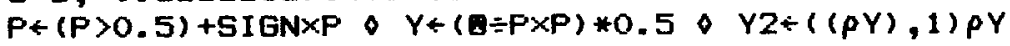

$\nabla$

$Z+5 I G N \times Y+(Y 2+5+C) \div Y Z 1-5+C$

$\nabla$ T*DF PTOT P;A;B;C;D;F;G;X;Y;S1;52;S3;S4;S5;56;57;S8;SIGN

[1] A DF CARRIES DEGREE (S) OF FREEDOM, P CARRIES PROBAB ILITY VALUE(S),

[2] A AND T RETURNS T VALUE (5); FOR EXAMPLE, FOF DF +1520 AND

[3] $A$ P $+0.050 .010 .95, T=6.314 \quad 3.365-1.725$

[4] DF*,DF \& Pt,P \& SIGN $1+-2 \times P>0.5 \circ \rightarrow(\cdots /(0<P) \wedge(1>F)) / 6$

[5]

[6]

[7]

[8]

[9]

[10]

$[11]$

$[12] S 4+(Y>0.05+A) / 2 \rho Y \diamond 55 *(\sim Y>0.05+A) / 2 \rho Y \diamond \rightarrow(0=+/ 54) / E L S E$
$[13] \quad X+-1 \times P T 0 Z P[S 3[54]] \times 0.5 \diamond G+X * 2 \diamond 58 *(D F[53[54]](5) / 2 \rho 54$

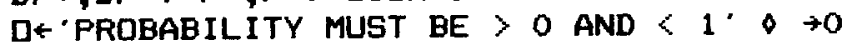

$P \leftarrow 2 \times(P>0.5)+5 I G N \times P \bullet \rightarrow((\rho D F) \neq \rho P) / 0$

$T \leftarrow(\rho P) \rho O \diamond 51 \div(D F=1) / 2 \rho D F \diamond 52 \div(D F=2) / 2 \rho D F$ o $S 3 \leftarrow(D F>2) / 2 \rho D F$

$P[51] \leftarrow P[S 1] \times 00.5 \& T[S 1] \div(20 P[51]) \div 10 P[S 1]$

$T[52] \div(-2+2 \div P[S 2] \times 2-P[S 2]) * 0.5 \bullet \rightarrow(0=+/ 53) / E N D \diamond A \leftarrow \div D F[53]-0.5$

$B+48 \div A * 2 \diamond C+96.36+A \times-16+A \times-98+20700 \times A \div B$

$D \div((A \times 00.5) * 0.5) \times D F[S 3] \times 1+(-3+94.5 \div C+B) \div B \diamond X \div D \times P[S 3] \diamond Y * X * 2 \div D F[53]$

$[14] \rightarrow(0=+/ 58) / N E X T \diamond C[54[58]]+C[54[58]]+0.3 \times(D F[53[54[58]]]-4.5) \times 0.6+X[58]$

[15] NEXT: C[54] $[\mathrm{C}[54]+\mathrm{B}[54]+X \times-2+X \times-7+X \times-5+X \times D[54] \times 0.05$

$[16]$

$G+X \times 1+((G \times-1)+-3+(94.5+G \times 36+G \times 6.3+G \times 0.4) \div C[54]) \div B[54] \diamond G * A[54] \times G * 2$ 
LISTING 1 (Continued)

[17] $S 6+\left(G>2 E^{-3}\right) / 2 \rho G \otimes S 7+\left({ }^{\sim} G>2 E^{-} 3\right) / 2 \rho G \otimes G[S 6] *^{-} 1+* G[S 6]$

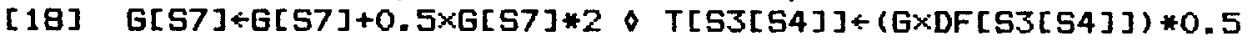

[19] ELSE: $\rightarrow(0=+/ 55) /$ END

[20] $F \div(-0.089 \times D[55])+-0.822+(6+D F[53[55]]) \div Y[55] \times D F[53[55]]$

[21] $F \leftarrow^{-} 1+(Y[55] \div 2 \times 4+D F[53[55]])+Y[55] \div(3 \times 2+D F[53[55]]) \times F$

[22] $F+(\div Y[55])+((1+D F[53[55]]) \div 2+D F[53[55]]) \times F$

[23] T[S3[S5]] $\leftarrow$ (DF[S3[S5] $] \times F) * 0.5$

[24] END: $T+T \times S I G N$

$\nabla$

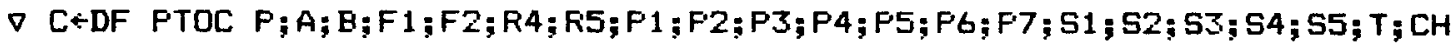

[1] A DF CARRIES DEGREE (S) OF FREEDOM, P CARRIES PROBABILITY VALUE (S),

[2] A AND C RETURNS CHI-SQUARE VALUE (S), FOR EXAMPLE, FOR DF $\div 1520$

[3] A AND $\mathrm{P} \div 0.050 .010 .95, \mathrm{C}=3.841 \quad 15.08610 .851$

[4] $D F \div, D F \diamond P \leftarrow, P \otimes \rightarrow((\rho D F) \neq \rho P) / 0 \diamond C H \div(\rho P) \rho O$

[5]

[6]

[7]

[8]

[9]

[10]

[11]

[12]

[13]

[14]

[15]

[16]

[17]

[18]

[19]

[20]

[21]

[22]

[23]

[24]

$\rightarrow(\wedge /(0<P) \wedge(1>P)) / 6 \bullet$ Q 4 PROBABILITY MUST BE $>0$ AND $<1 ; \diamond \rightarrow 0$

At $1.565326 E^{-3} \quad 1.060438^{-3}-6.95035599 E^{-} 3-0.01323293 \quad 0.02277679$

$A \div A,-8.986007 E^{-3}-0.01513904-1.450117 E^{-3} 2.53001 E^{-3} 5.169654 E^{-3}$

$A \leftarrow A,-0.01153761 \quad 0.01128186 \quad 0.02607083-0.2237368 \quad 9.780499 E^{-} 5$

$A+A,-8.426812 E^{-} 43.12558 E^{-3}-8.553069 E^{-3} \quad 1.348028 E^{-} 4$

$A \leftarrow A, 0.47139411 .0000886$

Bt $-0.01425296 \quad 0.01264616-5.88609 E^{-3} \quad 0.01400483-0.01091214$

$\mathrm{B}<\mathrm{B},-0.02304527-2.728484 \mathrm{E}^{-4} 3.135411 \mathrm{E}^{-3}-9.6996 \mathrm{~B}^{-} \mathrm{E}^{-} 3$

$\mathrm{B} \div \mathrm{B}, 0.013168720 .02618914-0.2222222 \quad 5.406674 \mathrm{E}^{-} 5 \quad 3.48378^{-} \mathrm{E}^{-5}$

$\mathrm{B}+\mathrm{B}, \quad-7.274761 \mathrm{E}^{-4} \quad 3.292181 \mathrm{E}^{-3}-8.729713 \mathrm{E}^{-3} \quad 0 \quad 0.47140451$

$S 1 \div(D F=1) / 2 \rho D F \diamond S 2 \div(D F=2) / 2 \rho D F \diamond S 3+(D F>2) / 2 \rho D F$

CH[S1] + PTOZ P[S1] $\times 0.5) * 2 \diamond$ CH[S2] $4-2 \times 0 P[52] \diamond \rightarrow(0=+/ 53) / E N D$

$F 1+D D F[53] \diamond T+P T O Z P[53] \diamond F 2+T \times F 1 * 0.5 \diamond 54 \div(D F[53]<2+L 4 \times 1 T) / 2 P T$

$S 5 \leftarrow(2 D F[S 3]<2+L 4 \times 1 T) / \imath \rho T \diamond \rightarrow(0=+/ S 4) / E L S E \diamond R 4+((\rho S 4), 1) \rho F 2[S 4]$

$P 1 \leftarrow R 4174 A$ o $P 2+R 41747$ QA $\& P 3+R 41-7+A$

CH[S3[S4] ] + DF [S3[S4] $] \times(P 3+F 1[S 4] \times P 2+F 1[S 4] \times P 1) * 3$

$E L S E: \rightarrow(0=+/ S 5) / E N D \diamond R 5 \leftarrow((p S 5), 1) \rho F 2[S 5] \diamond P 4+R 5124 B \diamond P 5+R 514 \uparrow 2 \Phi B$

$P 6+R 516460 B$ \& $P 7+R 51-84 B$

CH[S3[S5] ] $+D F[53[55]] \times(P 7+F 1[55] \times P 6+F 1[55] \times P 5+F 1[55] \times P 4) * 3$

$\nabla$

$\nabla F \div D F$ PTOF X;B;PQ;RK; TAIL;SUB

[1] A DF CARRIES DEGREES OF FREEDOM, X CARRIES PROBABILITY VALUE(S),

[2] A F RETURNS THE F VALUE (5); FOR EXAMPLE, DF $+32 p 153151030$

[3] $A$ AND $x+0.01 \quad 0.05 \quad 0.95$ RETURNS $F=16.258 \quad 3.288 \quad 0.371$

[4]

$[5]$

[6]

[7]

$\nabla$

$X+, X \diamond R K \leftarrow^{-} 1+1,-1+\rho D F \diamond \rightarrow(R K \neq \rho X) / 0 \diamond D F+(R K, 2) \rho D F \diamond S U B+X>0.5$ $\rightarrow(\wedge /(0<X) \wedge(1>X)) / 6 \diamond$ Q $*$ PRDBABILITY MUST BE $>0$ AND $<1 \cdot 0 \rightarrow 0$ $D F \div 5 U B \Phi D F \diamond$ TAIL $\div 1+-2 \times 5 U B \diamond X+5 U B+T A I L \times X \diamond P Q+1 \Phi D F \div 2 \diamond B \leftarrow P Q X I N B T A X$ $\nabla$ $F \leftarrow(D F[; 2] \times 1-B)=D F[; 1] \times B \diamond F+F * T A I L$

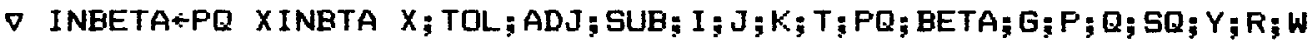

[1] A PQ CARRIES $P$ AND $Q$ VALUE( $S$ ), $X$ CARRIES PROBABILITY VALUE (S), INBETA

[2] A RETURNS VALUE(S) OF THE INUERSE INCOMPLETE BETA FUNCTION; FOR EXAMPLE,

[3] A PQ+3 $2 p 1022055030$ AND $X+0.00073400 .1110752,0.3170572$, YIELDS

[4] A INBETA $=0.40 \quad 0.70 \quad 0.60$

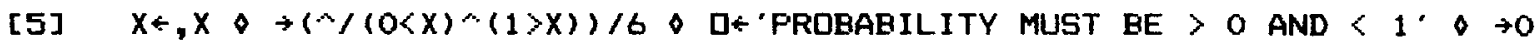

[6]

[7]

[8]

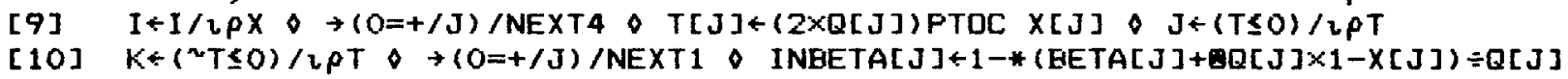

$R K+-1+1,-1+\rho P Q \otimes \rightarrow(R K \neq \rho X) / 0 \diamond P Q+(R K, 2) \rho P Q \diamond T+I N B E T A+(\rho X) \rho O$

$S U B \div X>0.5 \diamond P Q \div 5 U B \Phi P Q \diamond S I G N \div 1+-2 \times S U B \otimes X+5 U B+5 I G N \times X \otimes P+P Q[; 1]$

$Q+P Q[; 2] \diamond Y \in P T O Z X \diamond$ BETA+LNBETA $P Q \diamond I+(P \times Q) \geq P+Q \diamond J *(\sim I) / 2 \rho X$

[11] NEXT1 $: \rightarrow(0=+/ K) / N E X T 2 \diamond T[K] \div(-2+\{Q[K] \times 2)+P[K] \times 4) \div T[K] \diamond J+(T[K] \leq 1) / K$

[12] $K+(\sim T[K] \leq 1) / K$

[13] NEXT2: $\rightarrow(0=+/ J) /$ NEXT3 $\diamond$ INBETA[J] $*$ (BETA[J]+QX[J]XF[J] $) \div$ P[J]

[14] NEXT3: $\rightarrow(O=+/ K) /$ NEXT4 $\bullet$ INBETA[K $]+1-2 \div T[K]+1$ 
[15] NEXT4: $\rightarrow(0=+/ I) / I N I T I A L \diamond J \div(-3+Y[I] \times Y[I]) \div 6 \diamond K \div 2 \div(\div-1+2 \times P[I])+\div-1+2 \times Q[I]$

[16] $W+(J+(5 \div 6)-2 \div 3 \times K) \times(\div-1+2 \times Q[I])-\div-1+2 \times P[I] \diamond W+((1 Y[I] \times(J+K) * 0.5) \div K)-W$

[17] INBETA[I] $] \div P[I] \div P[I]+Q[I] \times * W+W$

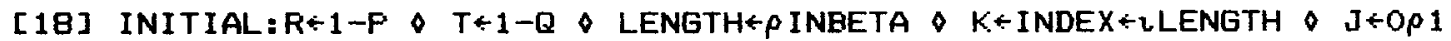

[19] YPREV 4 TEST $\div$ ADJ $\leftarrow L E N G T H P O \diamond S Q \div P R E V \div L E N G T H P 1$

[20] TOL $\div 10 *-1 \times L 13+(\div X * 0.2)+20 \div(P+P) * 2 \diamond$ INAETA[ (INBETAC1E -4$\left.) / I^{2} D E X\right] \div 1 E^{-4}$

[21] INBETA[ (INBETA>0.9999)/INDEX]+0.9999

[2Z] BEGIN: Y[K] PQ[K; ]BETAIN (INBETA[K])

[23] $Y[K]+(Y[K]-X[K]) \times * B E T A[K]+(R[K] \times B I N B E T A[K])+T[K] \times Q 1-I N B E T A[K]$

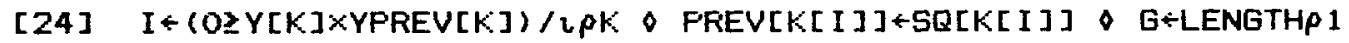

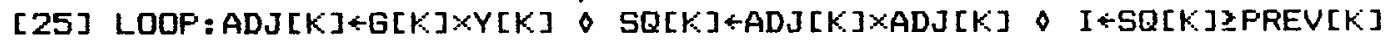

$[26] \rightarrow(0=+/ I) / C O N T$

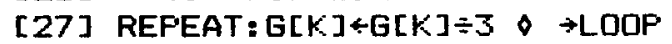

[2B] CONT: TEST $[K] \div I N B E T A[K]-A D J[K]$

[29] $I \leftarrow(T E S T[K]<0) \vee T E S T[K]>1 \diamond \rightarrow(0=+/ I) / C H E C K \diamond J \leftarrow J,(\sim I) / K \diamond K+I / K \diamond \rightarrow K E F E A T$ $[30]$ CHECK:K K K, J $\diamond K \leftarrow(P R E V[K]>$ TOL $[K]) / K \diamond \rightarrow(O=+/ K) / E N D$

$[31] K \leftarrow(T Q L[K]<Y[K] \times Y[K]) / K \diamond \rightarrow(0=+/ K) / E N D \diamond J \div O \rho 1 \diamond \quad I \div(T E S T[K]=0) \vee T E S T[K]=1$ $[32] \rightarrow(0=+/ I) / N E X T 5 \diamond J \leftarrow],(\sim I) / K \diamond K \leftarrow I / K \diamond \rightarrow R E F E A T$

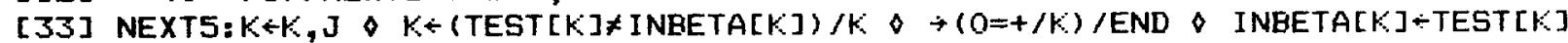

[34] YPREV $[K] \div Y[K] \diamond \rightarrow B E G I N$

[35] END: INBETA $\leftarrow$ SUB+SIGN $\times$ INBETA

$\nabla$

$\nabla$ INCBETAFPQ BETAIN X; I J J K; P; Q; PQ;PSQ; SUB; SIGN;NS; XX;AI;BETA;CX;RX; TOL

[1] A PQ CARRIES P AND $Q$ VALUE (S), $X$ CARRIES BETA DISTRIBUTIDN VALUE (S),

[2] A INCBETA RETURNS UPPER-TAIL PROBABILITY VALUE(S);

[3] A FOR EXAMPLE, $P Q+32 P 8610103835$ AND $X \div 0.710 .600 .57$, YIELDS

[4] A INCBETA $=0.85451210 .81390800 .8006772$

[5]

[6]

[7]

[B]

[9]

[ 10$]$

[11]

[12]

[13]

[ 14]

[15]

[16]

[17]

$X+, X \diamond R K{ }^{-}-141,-1+\rho P Q \diamond \rightarrow(R K \neq \rho X) / 0 \diamond T Q L+1 E^{-} B \diamond P Q+(R K, 2) \rho P Q \diamond P S Q++/ P Q$ $\rightarrow(\wedge /(0<X) \wedge(1>X)) / 7 \bullet$ D* BETA VALUES MUST BE $>0$ AND $<1 ; 0 \rightarrow 0$

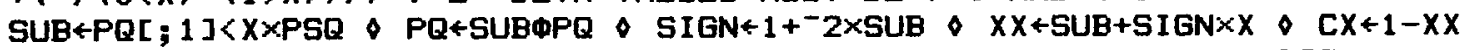

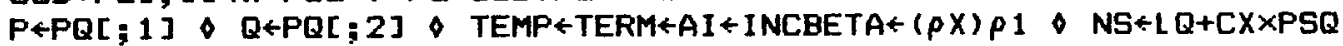
$R X \leftarrow X X \div C X \diamond J \leftarrow \imath \rho X \diamond K \leftarrow O P 1$

BEGIN: TEMP[J] Q[J]-AI[J] $\diamond I+(0=N S[J]) / J \diamond \rightarrow(0=+/ I) / N E X T \diamond R X[I] \leftarrow X X[I]$

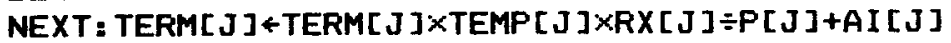

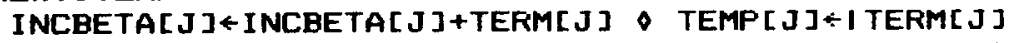

$J \leftarrow(($ TEMP $[J]>T Q L) \wedge($ TEMP $[J])>(T Q L \times I N C B E T A[J])) / J \diamond \rightarrow(0=+/ J) / C D N T$

$A I[J] \leftarrow A I[J]+1 \diamond N S[J] \leftarrow N S[J]-1 \diamond I \leftarrow 0>N S[J] \diamond \rightarrow(O=+/ I) / C D N T$

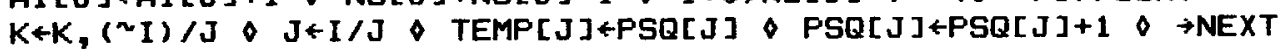

CONT $: J \leftarrow J, K \diamond \rightarrow(O=+/ J) / E N D \diamond K \leftarrow O \rho 1 \diamond \rightarrow B E G I N$ $\nabla$

INCBETA+SUB+SIGN $\times$ INCBETAX $(*((P \times E X X)+((Q-1) \times 8 C X)-B E T A)) \div F$

$\nabla$ BETA世LNBETA PQ; P;Q;PSQ; I J $; X ; Z ; R K ; C R I T ; B X ; C ; S U M ; L N G$

[1] $A$ PQ CARRIES $P$ AND $Q$ VALUES, BETA RETURNS THE NATURAL LOG OF THE

[2] A COMPLETE BETA FUNCTION; FOR EXAMPLE, PQ $\$ 2530$, BETA $=-38.2783$

[3] $R K t^{-} 141,-1+\rho P Q \diamond P Q \leftarrow(R K, 2) \rho P Q \diamond P S Q++/ P Q \diamond P \leftarrow P Q[; 1] \bullet Q \in P Q[2]$

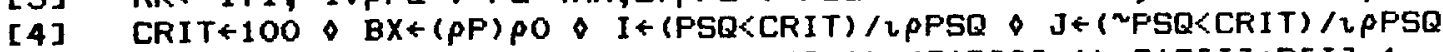

[5]

$[6]$

$\rightarrow(0=+/ I) / S T I R L I N G \bullet B X[I]+(8 ! Q[I]-1)+(8 ! P[I]-1)-8 ! Q[I]+P[I]-1$

STIRLING: $\rightarrow(0=+/ J) / E N D \diamond X \div P Q[J ;]$, PSQ[J] $\bullet Z \div \div \times x$

$C \leftarrow(\div 1680),(\div 1260),(\div 360), \div 12$

[7]

[8]

SUM $+(0.5 \times 2 \times 01)+(C[4]+Z \times C[3]+Z \times C[2]+Z \times C[1]) \div X$

[9] LNG $($ SUM $-X)+(X-\div 2) \times 8 X$ \& $B X[J]+L N G[; 1]+L N G[; 2]-L N G[; 3]$

[10] END: $B E T A+B X$

$\nabla$ 